\title{
Publisher's Note: Biological Magnetometry: Torque on Superparamagnetic Beads in Magnetic Fields \\ [Phys. Rev. Lett. 114, 218301 (2015)]
}

\author{
Maarten M. van Oene, Laura E. Dickinson, Francesco Pedaci, Mariana Köber, David Dulin, \\ Jan Lipfert, and Nynke H. Dekker
}

(A) (Received 8 June 2018; published 20 June 2018)

DOI: 10.1103/PhysRevLett.120.259901

This paper was published online on 27 May 2015 with an omission in the Acknowledgments on page 5. The last sentence of the Acknowledgments should read as "F. P. was supported by the European Research Council under the European Union's Seventh Framework Program (FP/2007-2013)/ERC Grant 306475." The Acknowledgments have been corrected as of 15 June 2018. The Acknowledgments are incorrect in the printed version of the journal. 